\title{
Children and young adults with parents with cancer: a population-based study
}

This article was published in the following Dove Press journal:

Clinical Epidemiology

12 March 2012

Number of times this article has been viewed

\author{
Astri Syse \\ Gjøril B Aas' \\ Jon H Loge ${ }^{2,3}$ \\ 'Cancer Registry of Norway, ${ }^{2}$ Oslo \\ University Hospital, ${ }^{3}$ University of \\ Oslo, Oslo, Norway
}

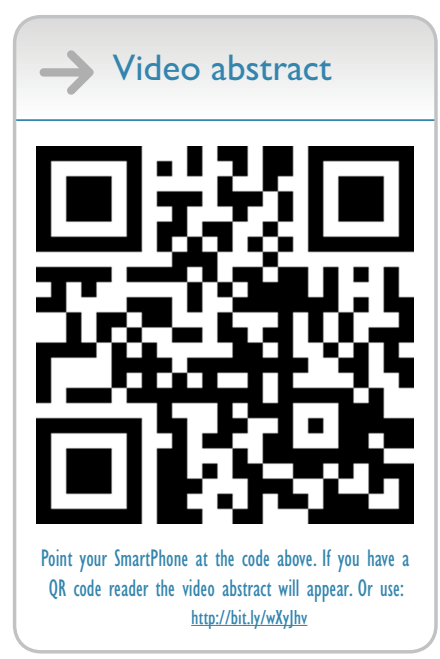

Correspondence: Astri Syse

Cancer Registry of Norway,

PO Box 5313 Majorstua,

$\mathrm{N}-0304$ Oslo, Norway

Tel +4747032304

Fax +47 $2245 \quad 1370$

Email astri.syse@kreftregisteret.no
Background: Today many people are choosing to have children later in life. Additionally, the use of sophisticated diagnostic tools and screening modalities has increased over recent years. Because of these factors, cancer is being diagnosed more frequently during the child-rearing years. Sociodemographic and cancer-related information on families and minor (0-18 years) and young adult (YA) (19-25 years) children experiencing parental cancer is scarce, but this information is vital for healthcare initiatives aimed toward those potentially adversely affected. Therefore, the aim of this study was to describe features of families and minor and YA children affected by parental cancer in a nationwide population.

Methods: Complete Norwegian birth cohort data were obtained from national registries. Descriptive prevalence and incidence statistics were collected for parents and minor and YA children. Logistic regression models were used to assess factors likely to influence parental death.

Results: Every year around $0.3 \%$ of all families with children under the age of 18 years encounter parental cancer, and $3.1 \%$ of minors and $8.4 \%$ of YAs have a parent who has been diagnosed with cancer. This study found skin, breast, testicular, and colorectal cancers were the most common forms of cancer diagnosed. The sociodemographic features of those affected were fairly similar to those of the general population. One in five children experienced parental death from cancer; parental death was more often paternal than maternal and was most common in parents diagnosed with leukemia or brain, colorectal, and lung tumors. Deaths are uncommon among parents without cancer.

Conclusion: Adequate assistance for minor and YA children affected by parental cancer requires knowledge of their number and characteristics. Parental cancer is more common than previously suggested: the annual incidence of parental cancer for children under 18 years of age is $0.3 \%$, whereas approximately $4 \%$ of children aged 0-25 years have or have had parents diagnosed with cancer, corresponding to a population prevalence of $1.4 \%$. Around $20 \%$ of these children experience parental death, and surveys of live respondents should account for this.

Keywords: incidence, neoplasm, parental cancer, prevalence, Norway

\section{Introduction}

Cancer survivors may face psychological, physical, social, and economic challenges as a consequence of their illness or treatment. ${ }^{1}$ About one-third of cancer patients are diagnosed at an age when they may possibly be caring for children - minor ( 0 -18 years) and/or young adult (YA) (19-25 years) children ${ }^{2}$ - and this means entire families can be affected by cancer. ${ }^{3,4}$ Children of parents affected by cancer are at risk for emotional and behavioral problems, although the majority cope well and are unlikely to develop such problems. ${ }^{5}$ The child's age, their stage of psychological development at the time 
of diagnosis and disease, and family characteristics modify the psychological burden of a parent's cancer upon the child. ${ }^{6}$ Still, up to $25 \%$ of children experiencing severe parental illness will experience lowered and/or anxious mood, sleep problems, poor concentration, or difficulties at school. ${ }^{7}$ In worst-case scenarios, severe parental illness may cause profound trauma with consequences for later psychosocial functioning.

Consequences of parental cancer have been shown to vary across cancer disease characteristics and by the role, age, and gender of the diagnosed parent. ${ }^{5,6,8-10}$ Reported discrepancies likely reflect the diversity of families that encounter parental cancer: the economic situation, employment status, and marital status of parents, as well as the number of children, place of residence, type of cancer, and prognosis, are all factors likely to mediate the impact of parental cancer upon their offspring. ${ }^{5,11,12}$ The impact has been found to further differ depending on whether the respondent is the child or the parent in the situation. ${ }^{9}{ }^{13}$ Finally, observed differences may in part be a consequence of suboptimal reliability and validity of the instruments available to assess children's coping abilities and needs. ${ }^{5,11,12,14}$

In recent years, communication challenges within families and the provision of support programs for involved children have come under increased focus. ${ }^{7}$ Still, many families' needs are frequently unmet, and the effort to address the psychosocial aspects of living with parental cancer is recognized as being in its infancy. ${ }^{15}$ As a first step, an assessment of the number and characteristics of affected children is important. Weaver et $\mathrm{al}^{16}$ recently performed an assessment of these factors in the United States, using selfreported survey data with a response rate of around $70 \%$. They used population weights to estimate that there are about 1.6 million cancer survivors residing with a total of about 2.9 million children under the age of 18 years in the United States today. ${ }^{16}$ As Weaver et $a{ }^{16}$ indicated, these numbers were estimated based upon self-reported data from one region only and the study lacked objective data, which may infer an underestimation of affected persons. Additionally, cancer types with short survival times were underrepresented and the study did not include children over 18 years of age. Estimates of the number of children experiencing parental death from cancer were not available, and calculations of incident cases, relevant for the shaping and development of services, were not performed. Therefore, Weaver et a ${ }^{16}$ requested complementary data to confirm their findings, as an account of the children and families affected by parental cancer is important to guide health care services assisting those potentially adversely affected. With this as a background, the present authors have summarized registry information on families and minor and YA children affected by parental cancer and parental death from cancer at a national level, in total as well as on an annual basis, in Norway.

\section{Methods}

Cancer patients and their children were identified through the unique eleven-digit personal identification number assigned to all inhabitants of Norway from 1960 onward. The Norwegian National Population Register provided information on date of birth, date of death or migration, dates of changes in marital status, and dates of birth of all children. Information regarding the education level of the parents was obtained from the Norwegian Registry of Education, and the Norwegian Directorate of Taxes provided information on their employment and yearly gross labor incomes. The Cancer Registry of Norway has registered all cancer cases nationwide since 1953. Mandatory reporting from clinicians and pathologists and mandatory use of death certificates ensures complete and excellent-quality data. ${ }^{17}$ The Cause of Death Registry provided cause of death data. Descriptive prevalence and incidence statistics were collated, and factors likely to influence parental death were assessed through logistic regression models.

Norway's population was calculated at 4.7 million as of January 1, 2008. ${ }^{18}$ Around $32 \%$ of the population aged between 17 and 70 years had at least one child under the age of 18 years, and $30 \%$ of the population aged between 35 and 70 years had at least one YA child (19-25 years of age). Persons with a history of cancer made up $4.3 \%$ and $3.3 \%$ of the female and male population, respectively. Of these, $80 \%$ were parents, but the majority of their children were older than the cut-off limit of 25 years of age. The present study included all persons 70 years or younger with children under the age of 26 years as of January 1, 2008; children were divided into two subgroups: minors and YAs. Next, two baseline prevalence cohorts were established: one comprised all minors in Norway as of January 1, 2008, and the other included all YAs. Prevalence analyses of sociodemographic characteristics (eg, age, gender, marital status, number of children, education level, and income) of parents and minor and YA children in families with and without parental cancer were undertaken, and parental disease (eg, cancer type, stage of cancer at diagnosis, and duration of illness) and death features were described in detail for the minor and YA children affected by parental cancer (Tables $1-4$ ). Logistic regression models were used to assess factors likely to influence parental death. From the two baseline prevalence cohorts, 2007 data 
Table I Prevalence data on Norwegian children with parents with cancer: common parental cancer diagnoses, stage of cancer at diagnosis, and the age of the child at diagnosis, as of January 2008

\begin{tabular}{|c|c|c|c|c|c|}
\hline \multirow[t]{2}{*}{ Variable } & \multirow[t]{2}{*}{ ICD- I 0 codes } & \multicolumn{2}{|c|}{ Cancer in mother } & \multicolumn{2}{|l|}{ Cancer in father } \\
\hline & & $\begin{array}{l}\text { Minor children } \\
{\left[N^{c}(\%)\right]}\end{array}$ & $\begin{array}{l}\text { YA children }^{\mathrm{b}} \\
{[\mathrm{N}(\%)]}\end{array}$ & $\begin{array}{l}\text { Minor children } \\
{[N(\%)]}\end{array}$ & $\begin{array}{l}\text { YA children } \\
{[N(\%)]}\end{array}$ \\
\hline \multicolumn{6}{|l|}{ Cancer site } \\
\hline Skin & C43-C44 & $3246(17.4)$ & $2217(12.6)$ & $2384(14.9)$ & $1892(13.5)$ \\
\hline Colorectal & $\mathrm{Cl}$-C2I & $1123(6.0)$ & $1572(8.9)$ & $1733(10.8)$ & $2350(16.8)$ \\
\hline Brain & C69-C72, D32-D33 & $1553(8.3)$ & II $22(6.4)$ & $1610(10.0)$ & I09| (7.8) \\
\hline Endocrine & $\mathrm{C} 73-\mathrm{C} 75$ & $1224(6.6)$ & $762(4.3)$ & $405(2.5)$ & $282(2.0)$ \\
\hline Leukemia & C90-C95 & $599(3.2)$ & $371(2.1)$ & $823(5.1)$ & $653(4.7)$ \\
\hline Non-Hodgkin disease & C82-C85, C95 & $56 I(3.0)$ & $466(2.6)$ & $856(5.3)$ & $877(6.3)$ \\
\hline Hodgkin disease & $\mathrm{C} 8 \mathrm{I}$ & $467(2.5)$ & I8I (I.0) & $809(5.0)$ & $324(2.3)$ \\
\hline Renal/bladder & C64-C68 & $300(1.6)$ & $314(1.8)$ & $963(6.0)$ & $1170(8.4)$ \\
\hline Lung & $\mathrm{C} 34$ & $284(1.5)$ & $518(2.9)$ & $614(3.8)$ & $1038(7.4)$ \\
\hline Head and neck & $\mathrm{C} 00-\mathrm{Cl} 4, \mathrm{C} 30-\mathrm{C} 32$ & $196(1.1)$ & $196(1.1)$ & $528(3.3)$ & $575(4.1)$ \\
\hline Soft tissue & C45-C49 & $162(0.9)$ & $104(0.6)$ & $188(1.2)$ & $13 \mid(0.9)$ \\
\hline Bone & C40-C4I & $130(0.7)$ & $66(0.4)$ & $185(1.2)$ & $89(0.6)$ \\
\hline Breast & C50 & $4950(26.6)$ & $6038(34.3)$ & N/A & N/A \\
\hline Cervical/uterine & C53-C55 & $2413(12.9)$ & $2276(12.9)$ & $\mathrm{N} / \mathrm{A}$ & $\mathrm{N} / \mathrm{A}$ \\
\hline Ovarian & C56 & $1247(6.7)$ & $1202(6.8)$ & $\mathrm{N} / \mathrm{A}$ & $\mathrm{N} / \mathrm{A}$ \\
\hline Testicular & C62-C63 & N/A & $\mathrm{N} / \mathrm{A}$ & $4043(25.2)$ & I569 (II.2) \\
\hline Prostate & $\mathrm{C} 61$ & $\mathrm{~N} / \mathrm{A}$ & $\mathrm{N} / \mathrm{A}$ & $600(3.7)$ & $1643(11.8)$ \\
\hline Other or unknown & C76-C80, C88 and others ${ }^{d}$ & $187(1.0)$ & $218(1.2)$ & $294(1.8)$ & $285(2.0)$ \\
\hline \multicolumn{6}{|l|}{ Stage of cancer at diagnosis } \\
\hline Local & N/A & $10030(53.8)$ & $8828(50.1)$ & $7870(49.1)$ & $6244(44.7)$ \\
\hline Regional & $\mathrm{N} / \mathrm{A}$ & $4623(24.8)$ & $4993(28.3)$ & $274 \mid(\mid 7.1)$ & $2540(18.2)$ \\
\hline Metastatic & N/A & $1399(7.5)$ & $1969(11.2)$ & $1779(11.1)$ & $2138(15.3)$ \\
\hline Unknowne & $\mathrm{N} / \mathrm{A}$ & $2590(13.9)$ & $1833(10.4)$ & $3645(22.7)$ & $3047(21.8)$ \\
\hline \multicolumn{6}{|l|}{ Age of child at diagnosis } \\
\hline $0 y$ & $\mathrm{~N} / \mathrm{A}$ & $656(3.5)$ & $201(1.1)$ & $680(4.2)$ & $185(1.3)$ \\
\hline $1-4 y$ & $\mathrm{~N} / \mathrm{A}$ & $3647(19.6)$ & $1252(7.1)$ & $2959(18.5)$ & $947(6.8)$ \\
\hline $5-9 y$ & N/A & $4877(26.2)$ & $2513(14.3)$ & $3566(22.2)$ & $1695(12.1)$ \\
\hline $10-14 y$ & $\mathrm{~N} / \mathrm{A}$ & $3377(18.1)$ & $2985(16.9)$ & $2274(14.2)$ & $2052(14.7)$ \\
\hline$\geq 15 y$ & N/A & $1714(9.2)$ & $9737(55.3)$ & $|32|(8.2)$ & $8048(57.6)$ \\
\hline Born $\mathrm{I}-4 \mathrm{y}$ after diagnosis & $\mathrm{N} / \mathrm{A}$ & $1547(8.3)$ & $387(2.2)$ & $1808(11.3)$ & $442(3.2)$ \\
\hline Born $\geq 5$ y after diagnosis & N/A & $2824(15.1)$ & $548(3.1)$ & $3427(21.4)$ & $600(4.3)$ \\
\hline
\end{tabular}

Notes: aMinor children are those aged between 0 and $18 \mathrm{y}$; ${ }^{\text {Y }}$ A children are those aged between 19 and $25 \mathrm{y}$; 'number of minor and YA children with parents with cancer; ${ }^{d}$ not otherwise specified; eincludes parental cancers with unknown stage and leukemias and around $50 \%$ of the lymphomas for which no stage was recorded.

Abbreviations: ICD-I0, International Classification of Diseases, I0th edition; N/A, not applicable; y, years; YA, young adult.

on the incidence of parental cancer diagnoses and the number of minor and YA children affected and not affected, as well as sociodemographic characteristics of parents and minor and YA children, were extracted to obtain estimates of the annual number and percentage of children affected by parental cancer (Table 5). All statistical analyses were performed using SAS software (v 9.2; SAS Institute Inc, Cary, NC). The statistical significance level was set at $5 \%$.

\section{Results}

\section{Scope of problem from a population perspective}

In 2007 a total of 84,202 women aged between 17 and 70 years had or had previously had cancer. Of these women,
11,374 had 18,642 children aged 18 years or younger. The total for men aged between 17 and 70 years was 67,554, with 9469 of these men having 16,035 children aged 18 years or younger. Similarly, 13,450 women with cancer were parents of 17,623 YA children, whereas 10,568 men with cancer were parents of 13,969 YA children. The total number of minors in Norway with one or two parents with a history of cancer as of January 1, 2008, was thus 34,250, representing $3.1 \%$ of minors in Norway as of this date. The corresponding number of YAs was 30,796, representing $8.4 \%$ of YAs as of January 1,2008 . The total number of children aged between 0 and 25 years with a parent with a history of cancer was 65,046 , corresponding to a population prevalence of $1.4 \%$. 
Table 2 Sociodemographic parental prevalence characteristics (as of January 2008) for children with and children without parents with cancer

\begin{tabular}{|c|c|c|c|c|}
\hline \multirow[t]{2}{*}{ Variable } & \multicolumn{2}{|l|}{ Cancer in mother } & \multicolumn{2}{|l|}{ Cancer in father } \\
\hline & $\begin{array}{l}\text { Minor children }{ }^{\mathrm{a}} \\
{[\% \text { cancer }(\% \text { GP) }]}\end{array}$ & $\begin{array}{l}\text { YA children }{ }^{\mathrm{b}} \\
{[\% \text { cancer }(\% \text { GP })]}\end{array}$ & $\begin{array}{l}\text { Minor children } \\
{[\% \text { cancer }(\% \text { GP) }]}\end{array}$ & $\begin{array}{l}\text { YA children } \\
{[\% \text { cancer }(\% \text { GP })]}\end{array}$ \\
\hline \multicolumn{5}{|l|}{ Year of diagnosis } \\
\hline Before 1980 & $2.3(\mathrm{~N} / \mathrm{A})$ & $2.3(\mathrm{~N} / \mathrm{A})$ & $3.4(\mathrm{~N} / \mathrm{A})$ & $3.2(\mathrm{~N} / \mathrm{A})$ \\
\hline $1980-1989$ & $7.1(\mathrm{~N} / \mathrm{A})$ & $9.2(\mathrm{~N} / \mathrm{A})$ & $9.3(\mathrm{~N} / \mathrm{A})$ & $10.5(\mathrm{~N} / \mathrm{A})$ \\
\hline $1990-1999$ & $27.5(\mathrm{~N} / \mathrm{A})$ & $33.0(\mathrm{~N} / \mathrm{A})$ & $31.1(\mathrm{~N} / \mathrm{A})$ & $28.9(\mathrm{~N} / \mathrm{A})$ \\
\hline $2000-2007$ & $63.1(\mathrm{~N} / \mathrm{A})$ & $55.5(\mathrm{~N} / \mathrm{A})$ & $56.2(\mathrm{~N} / \mathrm{A})$ & $57.4(\mathrm{~N} / \mathrm{A})$ \\
\hline \multicolumn{5}{|l|}{ Age at diagnosis } \\
\hline$<30 y$ & $24.6(\mathrm{~N} / \mathrm{A})$ & $8.2(\mathrm{~N} / \mathrm{A})$ & $25.5(\mathrm{~N} / \mathrm{A})$ & $7.4(\mathrm{~N} / \mathrm{A})$ \\
\hline $30-39 y$ & $40.3(\mathrm{~N} / \mathrm{A})$ & $23.7(\mathrm{~N} / \mathrm{A})$ & $33.3(\mathrm{~N} / \mathrm{A})$ & $16.5(\mathrm{~N} / \mathrm{A})$ \\
\hline $40-49 y$ & 30.9 (N/A) & $45.9(\mathrm{~N} / \mathrm{A})$ & $27.4(\mathrm{~N} / \mathrm{A})$ & $32.9(\mathrm{~N} / \mathrm{A})$ \\
\hline $50-59 y$ & $4.2(\mathrm{~N} / \mathrm{A})$ & $20.9(\mathrm{~N} / \mathrm{A})$ & $12.9(\mathrm{~N} / \mathrm{A})$ & $34.2(\mathrm{~N} / \mathrm{A})$ \\
\hline$>59 y$ & $0.0(\mathrm{~N} / \mathrm{A})$ & $\mathrm{I} .3(\mathrm{~N} / \mathrm{A})$ & $0.9(\mathrm{~N} / \mathrm{A})$ & $9.0(\mathrm{~N} / \mathrm{A})$ \\
\hline \multicolumn{5}{|l|}{ Vital status } \\
\hline Alive & $86.7(99.8)$ & $77.9(99.2)$ & $82.7(99.2)$ & $68.7(97.4)$ \\
\hline Dead & $13.3(0.2)$ & $22.1(0.8)$ & $17.3(0.8)$ & $31.3(2.6)$ \\
\hline \multicolumn{5}{|l|}{$\mathrm{Age}^{\mathrm{c}}$} \\
\hline$<45 y$ & $63.9(84.1)$ & $19.2(28.7)$ & $52.0(71.0)$ & $11.3(15.3)$ \\
\hline $45-49 y$ & $22.9(11.7)$ & $28.7(34.2)$ & $22.0(17.5)$ & $19.9(28.9)$ \\
\hline $50-54 y$ & $11.2(3.6)$ & $31.0(25.3)$ & $14.9(8.1)$ & $27.5(31.5)$ \\
\hline $55-59 y$ & $1.9(0.5)$ & I5.7 (9.4) & $8.0(2.7)$ & $22.5(16.7)$ \\
\hline $60-64$ y & $0.2(0.1)$ & $4.9(2.2)$ & $3.2(0.7)$ & $13.5(6.1)$ \\
\hline$>64 y$ & $\mathrm{~N} / \mathrm{A}$ & $0.5(0.2)$ & $\mathrm{N} / \mathrm{A}$ & $5.2(1.5)$ \\
\hline \multicolumn{5}{|l|}{ Education level ${ }^{c}$} \\
\hline Up to elementary school & $20.5(25.2)$ & $23.6(29.3)$ & $20.6(23.8)$ & $22.5(25.2)$ \\
\hline High school & $9.9(7.1)$ & $19.8(14.8)$ & $10.3(7.4)$ & $19.5(15.6)$ \\
\hline$<3 y$ of college & $25.5(27.0)$ & $19.9(23.4)$ & $29.4(32.9)$ & $23.9(27.4)$ \\
\hline $3-4 y$ of college & $36.6(33.7)$ & $31.5(28.3)$ & $27.7(25.6)$ & $23.6(22.4)$ \\
\hline$\geq 5$ y of college & $7.5(7.0)$ & $5.1(4.2)$ & I2.I (I0.3) & $10.6(9.4)$ \\
\hline \multicolumn{5}{|l|}{ Annual earnings ${ }^{c}$} \\
\hline$\$ 0-\$ 9999$ & $17.3(16.8)$ & $24.0(16.8)$ & $12.6(8.6)$ & $22.2(15.4)$ \\
\hline$\$ 10,000-\$ 19,999$ & $6.8(5.2)$ & $7.4(3.9)$ & $3.5(1.9)$ & $5.7(2.0)$ \\
\hline$\$ 20,000-\$ 39,999$ & $27.3(24.4)$ & $23.2(20.5)$ & $9.9(6.5)$ & $12.3(5.9)$ \\
\hline$\$ 40,000-\$ 59,999$ & $30.0(32.7)$ & $27.0(33.3)$ & $22.7(20.5)$ & $18.6(17.4)$ \\
\hline$\$ 60,000-\$ 79,999$ & $12.4(14.3)$ & $13.4(18.5)$ & $23.8(28.7)$ & $19.0(25.7)$ \\
\hline$\$ 80,000-\$ 99,999$ & $3.2(3.6)$ & $2.6(4.2)$ & 11.9 (14.9) & $8.9(14.3)$ \\
\hline$\geq \$ 100,000$ & $3.1(3.0)$ & $2.5(2.8)$ & I5.5 (I8.9) & I3.3 (19.3) \\
\hline \multicolumn{5}{|l|}{ Marital status ${ }^{c}$} \\
\hline Married & $64.4(59.4)$ & $68.7(66.8)$ & $65.2(6 I .1)$ & $72.5(69.8)$ \\
\hline Not married & $35.6(40.6)$ & $31.3(33.2)$ & $34.8(38.9)$ & $27.5(30.2)$ \\
\hline \multicolumn{5}{|l|}{ Children $(n)^{c}$} \\
\hline 1 & I3.4 (I8.9) & $8.7(7.2)$ & $13.9(19.3)$ & $7.7(7.3)$ \\
\hline 2 & $41.9(40.5)$ & $39.2(37)$ & $38.3(38.9)$ & $34.0(35.2)$ \\
\hline$\geq 3$ & $44.7(40.6)$ & $52.0(55.8)$ & $47.8(4 \mid .8)$ & $58.3(57.5)$ \\
\hline
\end{tabular}

Notes: a Minor children are those aged between 0 and $18 \mathrm{y}$; ' $Y A$ children are those aged between 19 and 25 ; ' as of January 2008 for those alive and at time of death for those deceased.

Abbreviations: GP, general population; N/A, not applicable; $y$, years; YA, young adult.

Altogether, 2075 families with 3481 children aged 18 years or younger experienced having a mother $(56 \%)$ and/or a father (44\%) diagnosed with cancer during 2007. This represents $0.3 \%$ of all families with minor children at one point in time, and is thus an estimate of the annual number of families affected, assuming stable birth and cancer incidence rates in the relevant age groups. The median age of the minor children was 8 years. A total of 2272 families with 2989 YA children were affected by parental cancer during 2007.

The number of minors who experienced parental death from cancer during 2007 was 665 , which corresponds to an annual percentage of $1.9 \%$. The percentage was somewhat 
Table 3 Distribution of children with parents with cancer having experienced parental cancer death by January 2008, by parental cancer site and stage of cancer at diagnosis

\begin{tabular}{|c|c|c|c|c|}
\hline \multirow[t]{2}{*}{ Variable } & \multicolumn{2}{|l|}{ Cancer in mother } & \multicolumn{2}{|l|}{ Cancer in father } \\
\hline & Minor children $^{a}(\%)^{c}$ & YA children ${ }^{\mathrm{b}}(\%)$ & Minor children (\%) & YA children (\%) \\
\hline \multicolumn{5}{|l|}{ Cancer site } \\
\hline Skin & 5.4 & 9.2 & 11.7 & 18.4 \\
\hline Colorectal & 42.5 & 43.3 & 45.2 & 51.1 \\
\hline Brain & 12.6 & 23.6 & 23.7 & 39.3 \\
\hline Endocrine & 2.6 & 5.0 & 7.7 & 13.8 \\
\hline Leukemia & 12.5 & 33.7 & 20.9 & 46.2 \\
\hline Non-Hodgkin disease & 10.7 & 21.9 & 12.9 & 30.2 \\
\hline Hodgkin disease & 4.5 & 18.2 & 5.6 & 20.4 \\
\hline Renal/bladder & 12.7 & 19.7 & 15.9 & 20.9 \\
\hline Lung & 58.5 & 73.4 & 64.2 & 80.3 \\
\hline Head and neck & 10.2 & 16.3 & 22.2 & 27.0 \\
\hline Soft tissue & 18.5 & 27.9 & 14.4 & 32.1 \\
\hline Bone & 11.5 & 22.7 & 18.4 & 41.6 \\
\hline Breast & 16.0 & 19.9 & N/A & $N / A$ \\
\hline Cervical/uterine & 8.2 & 15.2 & $\mathrm{~N} / \mathrm{A}$ & N/A \\
\hline Ovarian & 9.6 & 21.7 & $\mathrm{~N} / \mathrm{A}$ & N/A \\
\hline Testicular & N/A & N/A & 2.0 & 6.1 \\
\hline Prostate & $N / A$ & $\mathrm{~N} / \mathrm{A}$ & 9.2 & 10.5 \\
\hline Other or unknown ${ }^{d}$ & 38.5 & 56.0 & 37.4 & 50.5 \\
\hline \multicolumn{5}{|l|}{ Stage of cancer at diagnosis } \\
\hline Local & 5.6 & 10.3 & 8.3 & 16.6 \\
\hline Regional & 17.7 & 23.5 & 21.1 & 35.3 \\
\hline Metastatic & 61.7 & 72.0 & 56.3 & 74.8 \\
\hline Unknown ${ }^{\mathrm{e}}$ & 9.5 & 21.8 & 14.8 & 27.6 \\
\hline
\end{tabular}

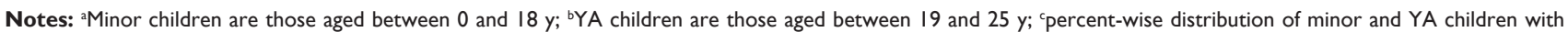
parents with cancer (I7/427 (4\%) minor children and 75/796 (9\%) YA children lost both parents after cancer and therefore the combined number of mothers and fathers

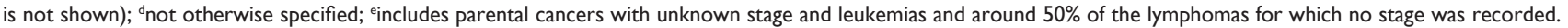
Abbreviations: N/A, not applicable; $y$, years; YA, young adult.

higher for fathers than for mothers $(2.0 \%$ versus $1.8 \%)$. The number of YAs who experienced parental death from cancer during 2007 was 805 , corresponding to an overall percentage of $2.5 \%$ (3.1\% for fathers versus $2.1 \%$ for mothers).

\section{Impact on minors in Norway with a parent once diagnosed with cancer}

Among the minors with a parent with (or with a history of) cancer in Norway, $54 \%$ had (or had previously had) a mother diagnosed with cancer and $47 \%$ had (or had previously had) a father diagnosed with cancer. A total of $33 \%$ of the minors with fathers with cancer and $23 \%$ of the minors with mothers with cancer were not yet born at the time of diagnosis (Table 1). The most common cancers among these parents were lymphomas and skin, brain, testicular, and breast cancers. With the exception of brain tumors, the prognosis is generally good for these cancer forms. Nevertheless, a total of $4.8 \%$ of these children experienced parental death from cancer, although this percentage is low compared with the children who had been born before the diagnosis and had lost their parent to cancer.
The remaining $67 \%$ and $77 \%$ of children were born either at $(4 \%)$ or before the time of their parent's cancer diagnosis (Table 1). The mean age of the minor children as of January 2008 was 12 years: 11 years if their father had cancer and 13 years if their mother had cancer. Their gender distribution was in line with the overall gender distribution in the population. More than half of the minors in Norway with a parent once diagnosed with cancer also had a parent diagnosed more than 5 years earlier. The most common maternal diagnoses experienced by minors were breast (27\%), skin (17\%), and cervical (13\%) cancer, whereas the most common paternal diagnoses were testicular $(25 \%)$, skin $(15 \%)$, and colorectal (11\%) cancer. The majority of parents were diagnosed with localized cancers.

One in seven minors with a parent once diagnosed with cancer had experienced parental death from cancer, whereas parental death is uncommon in the general population, being experienced by only about one in 300-400 (Table 2). Parental death was more common when fathers had cancer, and the most lethal diagnoses among fathers were lung, colorectal, and brain tumors (Tables 2-4). Among mothers, the most 
Table 4 Estimates from logistic regression models of characteristics impacting the likelihood of parental death from cancer among children with parents with cancer

\begin{tabular}{|c|c|c|c|c|}
\hline \multirow[t]{2}{*}{ Variable } & \multicolumn{2}{|l|}{ Minor children ${ }^{a}$} & \multicolumn{2}{|l|}{ YA children ${ }^{b}$} \\
\hline & $\begin{array}{l}\text { Cancer in father } \\
\text { OR }(95 \% \mathrm{Cl})\end{array}$ & $\begin{array}{l}\text { Cancer in mother } \\
\text { OR }(95 \% \mathrm{Cl})\end{array}$ & $\begin{array}{l}\text { Cancer in father } \\
\text { OR }(95 \% \mathrm{Cl})\end{array}$ & $\begin{array}{l}\text { Cancer in mother } \\
\text { OR }(95 \% \mathrm{Cl})\end{array}$ \\
\hline \multicolumn{5}{|l|}{ Cancer site } \\
\hline Other or unknown ${ }^{c}$ & $1.0(\mathrm{ref})$ & 1.0 (ref) & 1.0 (ref) & $\mathrm{I} .0$ (ref) \\
\hline Colorectal & $3.0(2.4-3.6)$ & $1.6(1.2-2.0)$ & $3.1(2.6-3.6)$ & I.I (0.9-I.4) \\
\hline Skin & $1.3(1.0-1.6)$ & $0.4(0.3-0.5)$ & $1.3(1.1-1.6)$ & $0.3(0.3-0.4)$ \\
\hline Brain & $4.5(3.6-5.6)$ & $1.2(1.0-1.6)$ & $5.2(4.3-6.3)$ & $1.5(1.2-1.9)$ \\
\hline Lymphoma & $0.5(0.4-0.6)$ & $0.3(0.2-0.4)$ & $1.2(1.0-1.4)$ & $0.4(0.3-0.5)$ \\
\hline Lung & $6.9(5.4-8.9)$ & $3.8(2.7-5.3)$ & II.5 (9.3-| 4.2$)$ & $4.7(3.5-6.2)$ \\
\hline Head and neck & $1.8(1.3-2.3)$ & $0.6(0.3-0.9)$ & $1.6(1.2-2.0)$ & $0.7(0.5-0.9)$ \\
\hline Renal/bladder & $1.4(1.1-1.8)$ & Other & $1.3(1.1-1.6)$ & Other \\
\hline Leukemia & $2.3(1.8-3.0)$ & Other & $4.6(3.7-5.8)$ & Other \\
\hline Testicular & $0.1(0.1-0.2)$ & N/A & $0.2(0.2-0.3)$ & $\mathrm{N} / \mathrm{A}$ \\
\hline Endocrine & Other & $0.1(0.1-0.2)$ & Other & $0.1(0.1-0.2)$ \\
\hline Breast & N/A & $0.7(0.6-0.9)$ & $\mathrm{N} / \mathrm{A}$ & $0.9(0.7-1.1)$ \\
\hline Cervical/uterine & $N / A$ & $0.4(0.3-0.5)$ & $\mathrm{N} / \mathrm{A}$ & $0.5(0.4-0.6)$ \\
\hline Ovarian & N/A & $0.2(0.2-0.3)$ & $\mathrm{N} / \mathrm{A}$ & $0.3(0.2-0.3)$ \\
\hline \multicolumn{5}{|l|}{ Stage of cancer at diagnosis } \\
\hline Local & $1.0(\mathrm{ref})$ & I.0 (ref) & 1.0 (ref) & I.0 (ref) \\
\hline Regional & $3.3(2.8-3.8)$ & $4.3(3.7-4.9)$ & $2.7(2.3-3.1)$ & $3.6(3.2-4.0)$ \\
\hline Metastatic & $21.4(18.0-25.5)$ & $25.5(21.1-30.7)$ & $17.0(14.6-19.7)$ & $35.5(29.9-42.1)$ \\
\hline Unknown & $2.0(1.7-2.3)$ & $1.8(1.5-2.2)$ & $1.8(1.6-2.1)$ & $2.8(2.4-3.3)$ \\
\hline \multicolumn{5}{|l|}{ Cancers (n) } \\
\hline 1 & $1.0(\mathrm{ref})$ & 1.0 (ref) & 1.0 (ref) & 1.0 (ref) \\
\hline$\geq 2$ & $2.1(1.6-2.7)$ & $1.6(1.3-2.0)$ & $1.9(1.6-2.3)$ & $1.9(1.7-2.3)$ \\
\hline \multicolumn{5}{|l|}{ Year of diagnosis } \\
\hline Before 1975 & 1.0 (ref) & $1.0(\mathrm{ref})$ & $\mathrm{I} .0$ (ref) & $1.0(\mathrm{ref})$ \\
\hline $1975-1984$ & $0.9(0.5-1.6)$ & $0.7(0.3-1.6)$ & $2.0(1.2-3.1)$ & $4.2(2 . I-8.4)$ \\
\hline $1985-1994$ & $2.2(1.3-3.7)$ & $3.5(1.7-7.5)$ & $2.8(1.8-4.4)$ & $7.7(4.0-15.2)$ \\
\hline 1995-2004 & $2.2(1.3-3.6)$ & $3.4(1.6-7.2)$ & $1.9(1.2-2.9)$ & $3.6(1.8-7.1)$ \\
\hline 2005 or later & $0.9(0.6-1.6)$ & $1.3(0.6-2.7)$ & $0.7(0.4-1.1)$ & $1.3(0.7-2.6)$ \\
\hline \multicolumn{5}{|c|}{ Age of parent at diagnosis: minor child (YA child) } \\
\hline$<20(<30)$ y & 1.0 (ref) & I.0 (ref) & I.0 (ref) & I.0 (ref) \\
\hline $20-29(30-39)$ y & $0.7(0.6-0.9)$ & $1.2(0.9-1.4)$ & I.I (0.9-1.3) & I.I (0.9-I.3) \\
\hline $30-39(40-49)$ y & $1.4(1.2-1.6)$ & $1.8(1.5-2.1)$ & $1.1(0.9-1.3)$ & $1.0(0.9-1.1)$ \\
\hline $40-49(50-59)$ y & $1.4(1.2-1.7)$ & $1.3(1.1-1.5)$ & I.I (0.9-1.3) & $0.8(0.7-0.9)$ \\
\hline$\geq 50(\geq 60)$ y & $1.4(1.1-1.7)$ & $0.8(0.6-1.1)$ & $1.2(1.0-1.5)$ & $0.9(0.6-1.4)$ \\
\hline \multicolumn{5}{|l|}{ SES of parent: marital status } \\
\hline Not married & $1.0(\mathrm{ref})$ & $1.0(\mathrm{ref})$ & 1.0 (ref) & $\mathrm{I} .0$ (ref) \\
\hline Married & $1.0(0.9-1.1)$ & $1.0(0.9-1.1)$ & $0.9(0.8 \mathrm{I}-0.99)$ & $1.0(0.91-1.10)$ \\
\hline \multicolumn{5}{|l|}{ SES of parent: children (n) } \\
\hline 1 & 1.0 (ref) & 1.0 (ref) & 1.0 (ref) & $1.0(\mathrm{ref})$ \\
\hline 2 & $0.6(0.5-0.8)$ & $0.8(0.7-0.9)$ & $0.7(0.6-0.8)$ & $0.8(0.7-0.9)$ \\
\hline 3 & $0.6(0.5-0.7)$ & $0.8(0.7-0.9)$ & $0.7(0.6-0.8)$ & $0.8(0.7-0.9)$ \\
\hline$\geq 4$ & $0.6(0.5-0.8)$ & $0.8(0.6-0.9)$ & $0.6(0.5-0.8)$ & $0.8(0.7-0.9)$ \\
\hline \multicolumn{5}{|l|}{ SES of parent: education level } \\
\hline Elementary school & 1.0 (ref) & $\mathrm{I} .0$ (ref) & 1.0 (ref) & 1.0 (ref) \\
\hline High school & $0.9(0.8-1.1)$ & I.I $(0.9-1.2)$ & $0.9(0.8-0.9)$ & $1.0(0.9-1.2)$ \\
\hline Some college & $0.8(0.7-0.9)$ & $0.7(0.6-0.8)$ & $0.7(0.6-0.8)$ & $0.6(0.5-0.7)$ \\
\hline Bachelor's degree & $0.6(0.5-0.7)$ & $0.6(0.5-0.7)$ & $0.7(0.6-0.8)$ & $0.7(0.6-0.8)$ \\
\hline Master's degree or higher & $0.7(0.6-0.9)$ & $0.5(0.4-0.7)$ & $0.8(0.7-0.9)$ & $0.7(0.5-0.8)$ \\
\hline SES of parent: income $/ \$ 10,000$ & $0.9(0.9-0.9)$ & $0.9(0.9-0.9)$ & $0.9(0.9-0.9)$ & $0.9(0.9-0.9)$ \\
\hline \multicolumn{5}{|l|}{ Birth year: minor child (YA child) } \\
\hline Before I 995 (1983) & $1.0(\mathrm{ref})$ & 1.0 (ref) & 1.0 (ref) & 1.0 (ref) \\
\hline $1995-1999(1984)$ & $0.8(0.7-0.9)$ & $0.8(0.7-0.9)$ & $0.9(0.8-1.1)$ & I.I (0.9-1.3) \\
\hline $2000-2004(1985)$ & $0.6(0.5-0.7)$ & $0.6(0.5-0.7)$ & I.0 (0.8-I.I) & $0.9(0.8-1.0)$ \\
\hline 2005 or later (1986 or later) & $0.2(0.2-0.3)$ & $0.3(0.3-0.4)$ & $0.8(0.7-0.8)$ & $0.9(0.8-0.9)$ \\
\hline
\end{tabular}

Notes: aMinor children are those aged between 0 and $18 \mathrm{y}$; ${ }^{\mathrm{C}} \mathrm{P}$ children are those aged between 19 and $25 \mathrm{y}$; includes unknown cancer sites and those not listed; dincludes missing.

Abbreviations: $\mathrm{Cl}$, confidence interval; N/A, not applicable; OR, odds ratio; SES, socioeconomic status; y, years; YA, young adult. 
Table 5 Incident characteristics of children with parents diagnosed with cancer (2007)

\begin{tabular}{|c|c|c|c|c|}
\hline \multirow[t]{2}{*}{ Variable } & \multicolumn{2}{|c|}{ Cancer in mother } & \multicolumn{2}{|l|}{ Cancer in father } \\
\hline & $\begin{array}{l}\text { Minor children } \\
{\left[\mathbf{N}^{\mathrm{a}}(\%)\right]}\end{array}$ & $\begin{array}{l}\text { YA children } \\
\text { [N (\%)] }\end{array}$ & $\begin{array}{l}\text { Minor children } \\
{[N(\%)]}\end{array}$ & $\begin{array}{l}\text { YA children } \\
\text { [N (\%)] }\end{array}$ \\
\hline \multicolumn{5}{|l|}{ Child's age at diagnosis } \\
\hline $0-1$ y & $39(2.0)$ & $\mathrm{N} / \mathrm{A}$ & $44(2.9)$ & N/A \\
\hline $\mathrm{I}-4 \mathrm{y}$ & $328(16.6)$ & $\mathrm{N} / \mathrm{A}$ & $268(17.8)$ & $\mathrm{N} / \mathrm{A}$ \\
\hline $5-9 y$ & $504(25.5)$ & $\mathrm{N} / \mathrm{A}$ & $350(23.2)$ & N/A \\
\hline $10-14 y$ & 731 (37.0) & $N / A$ & $531(35.3)$ & $N / A$ \\
\hline $15-18 y$ & $376(19.0)$ & $\mathrm{N} / \mathrm{A}$ & $313(20.8)$ & N/A \\
\hline $19-20 y$ & $\mathrm{~N} / \mathrm{A}$ & 399 (26.9) & $\mathrm{N} / \mathrm{A}$ & $367(24.1)$ \\
\hline $21-22 y$ & N/A & 428 (28.9) & N/A & $426(28.0)$ \\
\hline $23-25$ y & N/A & $654(44.2)$ & $\mathrm{N} / \mathrm{A}$ & $72 I(47.4)$ \\
\hline \multicolumn{5}{|l|}{ Cancer site } \\
\hline Skin & $219(11.1)$ & I $28(8.6)$ & $170(1 \mid .3)$ & |4| (9.3) \\
\hline Colorectal & $144(7.3)$ & $170(|1| .5)$ & $268(17.8)$ & $314(20.7)$ \\
\hline Brain & $165(8.3)$ & $88(5.9)$ & $126(8.4)$ & $81(5.3)$ \\
\hline Endocrine & $95(4.8)$ & $39(2.6)$ & $18(1.2)$ & $14(0.9)$ \\
\hline Leukemia & $46(2.3)$ & $34(2.3)$ & 91 (6.0) & $71(4.7)$ \\
\hline Non-Hodgkin disease & $56(2.8)$ & $57(3.8)$ & $76(5.0)$ & 7I (4.7) \\
\hline Hodgkin disease & $43(2.2)$ & $2(0.1)$ & $37(2.5)$ & $15(1.0)$ \\
\hline Renal/bladder & $23(1.2)$ & $28(1.9)$ & $138(9.2)$ & $|3|(8.6)$ \\
\hline Lung & $49(2.5)$ & $70(4.7)$ & $69(4.6)$ & $122(8.0)$ \\
\hline Head and neck & $18(0.9)$ & $13(0.9)$ & $70(4.6)$ & $51(3.4)$ \\
\hline Breast & 788 (39.8) & $608(4 I .1)$ & $\mathrm{N} / \mathrm{A}$ & $\mathrm{N} / \mathrm{A}$ \\
\hline Cervical/uterine & $186(9.4)$ & $136(9.2)$ & $\mathrm{N} / \mathrm{A}$ & $\mathrm{N} / \mathrm{A}$ \\
\hline Ovarian & $105(5.3)$ & $77(5.2)$ & N/A & N/A \\
\hline Testicular & N/A & $N / A$ & $186(12.4)$ & $4 \mid(2.7)$ \\
\hline Prostate & N/A & N/A & $201(13.3)$ & $435(28.6)$ \\
\hline Other or unknown ${ }^{d}$ & $4 I(2 . I)$ & $31(2.1)$ & $56(3.7)$ & $33(2.2)$ \\
\hline \multicolumn{5}{|c|}{ Stage of cancer at diagnosis } \\
\hline Local & 888 (44.9) & $562(37.9)$ & $507(33.7)$ & $498(32.8)$ \\
\hline Regional & $608(30.7)$ & $498(33.6)$ & $256(17.0)$ & $275(18.1)$ \\
\hline Metastatic & 177 (8.9) & $193(13.0)$ & $205(13.6)$ & $247(16.3)$ \\
\hline Unknown ${ }^{e}$ & $305(I 5.4)$ & $228(15.4)$ & $538(35.7)$ & $500(32.9)$ \\
\hline
\end{tabular}

Notes: a Minor children are those aged between 0 and $18 \mathrm{y}$; ${ }^{\text {} Y A}$ children are those aged between 19 and $25 \mathrm{y}$; ${ }^{\mathrm{c}}$ number of minor and YA children with parents with cancer; dsee Table I; eincludes parental cancers with unknown stage and leukemias and around $50 \%$ of the lymphomas for which no stage was recorded.

Abbreviations: N/A, not applicable; $y$, years; YA, young adult.

lethal diagnoses were lung, colorectal, soft tissue, and breast cancer. The highest death risks were observed for parents diagnosed with metastatic cancer. Parents of 427 minors $(1.2 \%)$ were diagnosed with more than one cancer form, corresponding to around $4.5 \%$ of mothers and $3.5 \%$ of fathers with cancer, and their risk of death was slightly elevated (Table 3).

Table 2 displays parental sociodemographic characteristics for minor and YA children with and without parents with cancer. The age distribution of the fathers with cancer spanned 20-63 years as of January 2008 (median 43.5 years). The age distribution of the mothers spanned 18-62 years (median 41.5 years). On average, parents with cancer were older than parents in the general population. A total of $31 \%$ of the parents had an education level no higher than a high school degree, but there was no difference in level of education between parents with and parents without cancer. Around $5.4 \%$ of the minors with mothers with cancer lived in households with annual incomes below the poverty margin defined by the Organization for Economic Cooperation and Development. ${ }^{19}$ The corresponding percentage for minors with fathers with cancer was $3.9 \%$. The household income distribution for minor children did not change significantly if their mother had cancer or not, whereas minor children of fathers with cancer generally appeared to live in households with income levels below that of the general cancer-free population. The vast majority of minors had parents who were married (only $17 \%$ had parents who were separated, divorced, or widowed). Marriage was less common in the general cancer-free population. The minors with parents with a history of cancer had from zero to ten siblings, but most commonly one or two; the average number of siblings was 1.3. 
The percentage of children with no siblings was lower in the cancer cohort than in the general population.

\section{Impact on YA children in Norway with a parent once diagnosed with cancer}

Cancer is a disease that becomes more common with increasing age, and around $93 \%$ of the YA children in this study were born before the time of the diagnosis of parental cancer. Around half had parents diagnosed within the last 5 years (Table 2). Maternal cancer was more prevalent than paternal cancer. The most common diagnoses in mothers were breast, cervical, and skin cancer, whereas diagnoses of colorectal, skin, prostate, and testicular cancer were frequent among fathers (Table 1). Nearly half the parents had localized cancers at the time of diagnosis, but the percentage of patients where the stage was unknown was clearly higher for fathers than mothers. One in four YAs with a parent once diagnosed with cancer had experienced parental death from cancer, whereas parental death is experienced by only one in 80 in the general population (Table 4). Death from cancer was more common in fathers than in mothers, and the most lethal diagnoses were lung and colorectal cancer. As expected, the highest probabilities of death were observed for parents diagnosed with metastatic cancer (Tables 3 and 4). A total of 796 YAs (2.6\%) had parents diagnosed with two or more cancer forms $(5.8 \%$ of mothers and $4.8 \%$ of fathers), and the risk of death for these parents was elevated.

Table 2 displays parental sociodemographic characteristics for YAs with and without parents with cancer. The age distribution of the fathers with cancer spanned 21-70 years of age as of January 2008 (median 52.8 years), whereas for mothers the age range spanned 20-70 years (median 49.3 years). Parents with a history of cancer were significantly older, on average, than parents without cancer. Around $43 \%$ of the parents had an education level no higher than a high school degree - this is comparable with general population figures. However, a higher percentage of parents with cancer had education levels corresponding to a bachelor degree or higher. On average, earnings were somewhat lower for parents (regardless of gender) with cancer. The majority of parents with cancer were married (71\%), and this figure is comparable with that for parents without cancer. The number of siblings for YA children ranged from zero to twelve, but they most commonly had one or two; the average number of siblings was 1.1. The gender distribution of YAs was fairly equal, and their median age as of January 2008 was 21 years.

\section{Annual impact of parental cancer on minor and YA children}

Altogether, 2075 families with 3481 children aged 18 years or younger experienced having the mother (56\%) and/or the father (44\%) diagnosed with cancer during 2007. The most common parental cancer diagnoses were breast, prostate, colorectal, skin, cervical, and testicular cancer, and the majority of cancers were localized (Table 5). A total of $7 \%$ of minors experienced maternal cancer diagnosis and maternal death from cancer during the same year, whereas the total for the children of fathers with cancer was $13 \%$. Altogether, 2272 families with 2989 YA children were affected, and the corresponding figures for cancer diagnosis and death from cancer within 1 year were $11 \%$ and $17 \%$, respectively.

The size of the families varied, from one parent and one child through to two parents and eleven children, but the two-parent family with two to three children was the most common (60\%), comparable with general population figures. Nearly $60 \%$ of the parents were married, and $20 \%$ were widowed, divorced, and/or separated adults, in line with general population estimates.

\section{Discussion}

This nationwide study shows that the annual incidence of parental cancer for minors is $0.3 \%$. At any one point in time, approximately $3 \%$ of minors and $8 \%$ of YA children have or have had parents once diagnosed with cancer, corresponding to a population prevalence of $1.4 \%$.

\section{Influential sociodemographic factors and existing research}

The vast majority of minor and YA children have taken part in a parent's cancer journey, and are likely influenced by and involved in their parent's illness and treatment. ${ }^{11,15,20}$ Around $16 \%$ of the minor and YA children had not yet been born at the time of their parent's diagnosis. These children were less likely to be affected by parental cancer, although long-term or late effects may be present in future years; additionally, fear of recurrence may influence family life. Five percent of these children lost their parent to cancer; however, this percentage is low compared with the children who had been born before the diagnosis and had lost their parent to cancer.

The demographic and socioeconomic characteristics of parents with and parents without cancer were fairly similar with respect to marital status and number of children. This was expected, as cancer has only a modest effect on parental marriage or divorce probabilities, ${ }^{21,22}$ and although the likelihood 
of parenthood is reduced after cancer, ${ }^{23}$ the majority of cancer survivors have completed their childbearing by the time of diagnosis. Compared with general population figures, a higher percentage of parents with cancer had earned a bachelor's degree or higher. This may make the children of these parents less at risk than the children of parents with other chronic diseases more closely associated with lower levels of education, as education has been shown to increase health literacy and to facilitate open dialogues. ${ }^{24-26}$ An understanding of the health and welfare systems may help navigation and thus improve coping and reduce stress, benefitting the children involved. However, incomes were on average lower for parents with cancer. This may reflect the older average age of the parents with cancer and thus the probability of being retired. ${ }^{27}$ However, it may also reflect work limitations associated with cancer. ${ }^{27,28}$ Although single parents, most commonly mothers, make up only a small proportion $(5 \%-10 \%)$ of parents in Norway, their children may be particularly vulnerable, ${ }^{29}$ and research targeting this specific subgroup may be warranted.

Some studies report adverse consequences for children affected by parental cancer, and the children's age and gender has been found to influence the type and degree of problems they experience. ${ }^{6,9,13}$ However, other studies do not report significant adverse consequences for children. ${ }^{3,30}$ Younger children may not fully comprehend the significance of parental cancer, and thus they may be less affected emotionally than older minors and YAs. ${ }^{9}$ Older siblings may support one another, but they may also experience a role shift if they need to assist more with household chores and/or assist with care of the ill parent. ${ }^{8}$ How this influences the children and parents involved is debatable, but studies report both positive and negative consequences. . $^{5,8,11,12,31}$

Between one in seven and one in four minor and YA children with parents with a history of cancer experience parental death, whereas parental death is uncommon in the general population. Children experience maternal cancer more often than paternal cancer, but they are more likely to lose a father than a mother to cancer. Studies on how families and minor and YA children cope with terminal care and parental death suggest that parents tend to underestimate the adverse consequences as they are perceived by the children involved, and that open communication and maintaining "normality" are important strategies for optimizing family functioning. ${ }^{32,33}$ Several studies report that the gender of the parent with cancer has implications for the psychosocial and emotional outcomes of the children involved. Most studies suggest that children respond worse to maternal than paternal cancer, ${ }^{13,34,35}$ perhaps in line with traditional caregiver roles. However, one study reports that the consequences are worse for children of fathers with cancer - this may reflect the poorer prognosis for fathers. ${ }^{6}$ The gender of the children involved is also reported to affect the prevalence and severity of problems reported. ${ }^{5,11}$ As the gender distribution of children is fairly equal and as it is similar for parents with and parents without cancer, the authors do not discuss this further here.

The authors' main findings on minor children of cancer survivors are in accordance with US estimates from Weaver et al, ${ }^{16}$ but the current authors present more detailed data on stage at diagnosis and death, and information on YA children is also included. Nonetheless, some important differences are notable: around $54 \%$ of the minors who had a parent with a history of cancer had experienced maternal cancer in the current study, whereas this was observed for $79 \%$ of the children in Weaver et al's study. ${ }^{16}$ These differences may be explained in part by the relatively high lethality of paternal cancer, as only living parents were included in the US study ${ }^{16}$ Selection and/or information bias may also have affected the US survey estimates. ${ }^{36}$ Women have been found more likely to respond to surveys and to underreport their cancer diagnosis less frequently than men. ${ }^{37,38}$ Nonetheless, the observed discrepancy underscores the need to confirm survey findings by using additional data sources and the importance of undertaking studies across different cultures.

\section{Generalizability of implications of findings}

Data on the entire Norwegian population of minor and YA children as of January 2008 has been utilized, and no earlier investigation has employed such detailed material on such a large scale. The use of national registry data minimizes bias, and the accuracy of population-based estimates resulting from survey data has thus been addressed. Furthermore, the results may be of general interest, as Norwegian cancer incidence and mortality trends and general family behaviors are comparable with those of other developed countries, including the United States. ${ }^{2,18,39}$ However, Norway is a welfare state, and cancer care is available to all, free of charge. In addition, multiple welfare benefits are endorsed to compensate for declines in earnings. This implies that comparisons of the possible influence of sociodemographic factors are more difficult crossculturally. However, it seems likely that findings may apply to countries with similar health and welfare options (eg, Canada and other Nordic and Western European countries).

As the data for this study are linked through the children, only the individual and combined characteristics of mothers and fathers can be addressed, not the family as a system per se. Knowledge of actual household members would have 
improved the estimate of, for instance, the impact of marital status and earnings. It would also have facilitated a better comparison with the existing US population estimates. ${ }^{16}$

Many of the factors useful in assessing needs and planning interventions to assist affected families have been assessed in a complete national population, and the authors have further shown that survey data may underestimate the number of children affected by parental cancer, as parental death, especially of fathers, is a relatively common phenomenon. In January 2010, a Norwegian law was implemented mandating minor children of severely ill parents be included as targets for support within the national health care system. ${ }^{40}$ The white papers preceding the law specifically mentioned cancer as one of several illnesses covered, and Norwegian cancer care is now obliged to provide services to assess the presence of minor children, assess their needs, and initiate interventions. ${ }^{41,42}$ Oncologic departments have been required to dedicate one specific health care practitioner to ensuring the presence of minor children is documented in cancer patients' journals and that the needs of involved children are assessed. ${ }^{1}$ This health practitioner is further obligated by law to contact social services or other relevant personnel if deemed necessary, irrespective of parental consent. ${ }^{40}$

Around $8 \%$ of YAs have or have had a parent diagnosed with cancer, corresponding to a population prevalence of $1.4 \%$. To the best of the authors' knowledge, this study is the first to contribute detailed information of YA children afflicted by parental cancer. Although YAs are independent in the legal sense, many nonetheless depend on parental support - physically, psychologically, socially, and economically. ${ }^{43}$ This dependence may alter when a parent is diagnosed with cancer, and caregiver burdens may in some cases also be reversed. ${ }^{44-46}$ From a health care perspective, the needs of and follow-up required for YA children in transitional phases will perhaps be different than for minor children, and research to address this difference is warranted.

Finally, information that has not been available previously on cancer prognosis and death from cancer has now been provided. Death is the most serious and dreaded consequence of parental cancer for the minor and YA children involved, ${ }^{5,11}$ and knowledge of the risk of parental death is useful when interacting with children and their parents in cases of parental cancer.

\section{Conclusion}

The annual incidence of parental cancer for minor children was $0.3 \%$, whereas the prevalence of having or having had a parent with cancer was $3 \%$ among minor children and $8 \%$ among YA children, corresponding to a population prevalence of $1.4 \%$. The most common diagnoses experienced by minor and YA children were skin, breast, testicular, and colorectal cancer; this distribution differs somewhat from the cancer distribution in the general population because of the age distribution of parents. ${ }^{2}$ One in five minor and YA children experience parental death from cancer - lung, colorectal, and brain tumors are most lethal. The proportion of children affected by cancer remains similar regardless of whether it is the mother or the father with cancer, but children are much more likely to experience paternal than maternal death. With this as the history, surveys of live respondents likely underestimate the number of children affected by parental cancer, especially fathers.

Adequate assistance for minor and YA children affected by parental cancer requires knowledge of their number and characteristics, as well as the characteristics of their parents. In recent years, communication challenges within families and the provision of support programs for involved children have come under increased focus. ${ }^{7}$ Nevertheless, many families' needs are frequently unmet, and the effort to address the psychosocial aspects of living with parental cancer is recognized as being in its infancy. ${ }^{15}$ Changes in the Norwegian Health Personnel Law in effect from January 2010 mandate health practitioner assessment of the presence of children when adults are diagnosed with cancer, assessment of the needs of involved children, and instigation of interventions to meet the needs of these children. ${ }^{40-42}$ This study has provided information at a population level to aid in this work, and the knowledge gained may thus help to understand what is required for the adequate assessment of needs and the proper assistance and follow-up of potentially vulnerable children experiencing parental cancer, particularly those facing parental death.

\section{Acknowledgment}

This research was supported by a grant from the Norwegian Research Council.

\section{Disclosure}

The authors report no conflicts of interest in this work.

\section{References}

1. Hewitt M, Stovall E, Greenfield S. From Cancer Patient to Cancer Survivor: Lost in Transition. Washington, DC: National Academies Press; 2006.

2. Ferlay J, Shin HR, Bray F, Foreman D, Mathers C, Parkin DM. GLOBOCAN 2008, Cancer Incidence and Mortality Worldwide: IARC CancerBase No 10 [Internet]. Lyon, France: International Agency for Research on Cancer; 2010. Available from: http://globocan.iarc.fr. Accessed September 9, 2010. 
3. Schmitt F, Santalahti P, Saarelainen S, Savonlahti E, Romer G, Piha J. Cancer families with children: factors associated with family functioning: a comparative study in Finland. Psychooncology. 2008; 17(4):363-372.

4. Vannatta K, Ramsey RR, Noll RB, Gerhardt CA. Associations of child adjustment with parent and family functioning: comparison of families of women with and without breast cancer. J Dev Behav Pediatr. 2010;31(1):9-16.

5. Osborn T. The psychosocial impact of parental cancer on children and adolescents: a systematic review. Psychooncology. 2007;16(2):101-126.

6. Thastum M, Watson M, Kienbacher C, et al. Prevalence and predictors of emotional and behavioural functioning of children where a parent has cancer: a multinational study. Cancer. 2009;115(17):4030-4039.

7. Moore CW, Rauch PK. Addressing the needs of children when a parent has cancer. In: Holland JC, editor. Psycho-Oncology. 2nd ed. Oxford: Oxford University Press; 2010:527-531

8. Visser A, Huizinga GA, Hoekstra HJ, van der Graaf WT, HoekstraWeebers JE. Parental cancer: characteristics of parents as predictors for child functioning. Cancer. 2006;106(5):1178-1187.

9. Visser A, Huizinga GA, Hoekstra HJ, et al. Emotional and behavioural functioning of children of a parent diagnosed with cancer: a crossinformant perspective. Psychooncology. 2005;14(9):746-758.

10. Rauch PK, Muriel AC, Cassem NH. Parents with cancer: who's looking after the children? J Clin Oncol. 2003;21(9 Suppl):S117-S121.

11. Visser A, Huizinga GA, van der Graaf WT, Hoekstra HJ, HoekstraWeebers JE. The impact of parental cancer on children and the family: a review of the literature. Cancer Treat Rev. 2004;30(8): 683-694.

12. Semple CJ, McCance T. Parents' experience of cancer who have young children: a literature review. Cancer Nurs. 2010;33(2):110-118.

13. Welch AS, Wadsworth ME, Compas BE. Adjustment of children and adolescents to parental cancer: parents' and children's perspectives. Cancer. 1996;77(7):1409-1418.

14. Pearce NJ, Sanson-Fisher R, Campbell HS. Measuring quality of life in cancer survivors: a methodological review of existing scales. Psychooncology. 2008;17(7):629-640.

15. Lewis FM. Parental cancer and dependent children: selected issues for future research. Psychooncology. 2007;16(2):97-98.

16. Weaver KE, Rowland JH, Alfano CM, McNeel TS. Parental cancer and the family: a population-based estimate of the number of US cancer survivors residing with their minor children. Cancer. 2010;116(18):4395-4401.

17. Larsen IK, Småstuen M, Johannesen TB, et al. Data quality at the Cancer Registry of Norway: an overview of comparability, completeness, validity and timeliness. Eur J Cancer. 2009;45(7):1218-1231.

18. Statistics Norway. Population. Oslo: Statistics Norway; [updated 2009]. Available from: http://www.ssb.no/befolkning_en/. Accessed April 5, 2011.

19. Organization for Economic Cooperation and Development (OECD). Child poverty. Paris: OECD; [updated 2010]. Available from: http://www.oecd.org/dataoecd/52/43/41929552.pdf. Accessed July 6, 2011.

20. Grabiak BR, Bender CM, Puskar KR. The impact of parental cancer on the adolescent: an analysis of the literature. Psychooncology. 2007;16(2):127-137.

21. Syse A. Does cancer affect marriage rates? J Cancer Surviv. 2008;2(3):205-214.

22. Syse A, Kravdal O. Does cancer affect the divorce rate? Demographic Res. 2007;16(15):469-492.

23. Syse A, Kravdal O, Tretli S. Parenthood after cancer: a population-based study. Psychooncology. 2007;16(10):920-927.

24. Garcia SF, Hahn EA, Jacobs EA. Addressing low literacy and health literacy in clinical oncology practice. J Support Oncol. 2010;8(2):64-69.
25. Davis TC, Williams MV, Marin E, Parker RM, Glass J. Health literacy and cancer communication. CA Cancer J Clin. 2002;52(3):134-149.

26. Health literacy: report of the Council on Scientific Affairs; Ad Hoc Committee on Health Literacy for the Council on Scientific Affairs, American Medical Association. JAMA. 1999;281(6):552-557.

27. Mehnert A. Employment and work-related issues in cancer survivors. Crit Rev Oncol Hematol. 2011;77(2):109-130.

28. Syse A, Tretli S, Kravdal O. Cancer's impact on employment and earnings: a population-based study from Norway. J Cancer Surviv. 2008;2(3):149-158.

29. Annunziato RA, Rakotomihamina V, Rubacka J. Examining the effects of maternal chronic illness on child well-being in single parent families. J Dev Behav Pediatr. 2007;28(5):386-391.

30. Lindqvist B, Schmitt F, Santalahti P, Romer G, Piha J. Factors associated with the mental health of adolescents when a parent has cancer. Scand J Psychol. 2007;48(4):345-351.

31. Turner J. Children's and family needs of young women with advanced breast cancer: a review. Palliat Support Care. 2004;2(1):55-64.

32. Christ GH, Siegel K, Sperber D. Impact of parental terminal cancer on adolescents. Am J Orthopsychiatry. 1994;64(4):604-613.

33. Kennedy VL, Lloyd-Williams M. How children cope when a parent has advanced cancer. Psychooncology. 2009;18(8):886-892.

34. Schmitt F, Piha J, Helenius H, et al. Multinational study of cancer patients and their children: factors associated with family functioning. J Clin Oncol. 2008;26(36):5877-5883.

35. Compas BE, Worsham NL, Epping-Jordan JE, et al. When mom or dad has cancer: markers of psychological distress in cancer patients, spouses, and children. Health Psychol. 1994;13(6):507-515.

36. Goodman A, Gatward R. Who are we missing? Area deprivation and survey participation. Eur J Epidemiol. 2008;23(6):379-387.

37. Nord C, Mykletun A, Fosså SD. Cancer patients' awareness about their diagnosis: a population-based study. J Public Health Med. 2003;25(4):313-317.

38. Nord-Trøndelag Health Study (HUNT). Response rates in HUNT. Trondheim: HUNT, Norwegian University of Science and Technology; [updated 2010]. [Norwegian]. Available from: http://www.ntnu.no/hunt/ oppmote. Accessed April 3, 2011.

39. PRB.org [homepage on the Internet]. Washington, DC: Population Reference Bureau; [updated 2010]. Available from: http://www.prb. org/. Accessed July 3, 2011.

40. Lovdata. Norwegian law re. health workers. Oslo: Lovdata, Norwegian laws; [updated 2010]. [Norwegian]. Available from: http://www. lovdata.no/cgi-wift/ldles?doc=/all/nl-19990702-064.html. Accessed August 8, 2011.

41. Norwegian Directorate of Health. Rundskriv IS-5/2010: Barn som pårørende [Children as significant others]. Norwegian Department of Health; [updated 2010]. [Norwegian]. Available from: http://www. barnsbeste.no. Accessed September 7, 2011.

42. Norwegian Department of Health. Ot.prp. nr.85 (2008-2009). Re. changes in the law re. health practitioners: follow-up of children as significant others. Oslo: Lovdata, Norwegian Laws; [updated 2010]. Accessed August 9, 2011.

43. Clark W. Delayed transitions of young adults. Statistics Canada [serial on the Internet]. Sep 2007 [updated 2008]; (84):13-21. Available from: http://www.statcan.gc.ca/pub/11-008-x/2007004/pdf/10311-eng.pdf. Accessed August 5, 2011.

44. Raveis VH, Pretter S. Existential plight of adult daughters following their mother's breast cancer diagnosis. Psychooncology. 2005;14(1):49-60.

45. Lackey NR, Gates MF. Adults' recollections of their experiences as young caregivers of family members with chronic physical illnesses. J Adv Nurs. 2001;34(3):320-328.

46. Gates MF, Lackey NR. Youngsters caring for adults with cancer. Image J Nurs Sch. 1998;30(1):11-15. 


\section{Publish your work in this journal}

Clinical Epidemiology is an international, peer-reviewed, open access journal focusing on disease and drug epidemiology, identification of risk factors and screening procedures to develop optimal preventative initiatives and programs. Specific topics include: diagnosis, prognosis, treatment, screening, prevention, risk factor modification, systematic

Submit your manuscript here: http://www.dovepress.com/clinical-epidemiology-journal reviews, risk \& safety of medical interventions, epidemiology \& biostatical methods, evaluation of guidelines, translational medicine, health policies \& economic evaluations. The manuscript management system is completely online and includes a very quick and fair peer-review system, which is all easy to use. 